\title{
Steady flow-induced instability of a partially embedded pipeline: Pipe-soil interaction mechanism
}

\author{
Fu-Ping Gao ${ }^{a, *}$, Shu-Ming Yan ${ }^{\mathrm{a}, \mathrm{b}}$, Bing Yang ${ }^{\mathrm{a}}$, Cheng-Cai Luo ${ }^{\mathrm{c}}$ \\ ${ }^{a}$ Key Laboratory for Hydrodynamics and Ocean Engineering, Institute of Mechanics, Chinese Academy of Sciences, Beijing 100190, China \\ ${ }^{\mathrm{b}}$ Crossing Department, China Petroleum Pipeline Engineering Corporation, Langfang 065000, China \\ ${ }^{\mathrm{c}}$ Centre for Offshore Foundation Systems, The University of Western Australia, Crawley, WA 6009, Australia
}

\section{A R T I C L E I N F O}

\section{Available online 28 September 2010}

Keywords:

Submarine pipeline

On-bottom stability

Pipe-soil interaction

Ocean currents

Sandy seabed

\begin{abstract}
A B S T R A C T
The steady flow-induced instability of a partially embedded pipeline involves a complex process of pipe-soil interaction. In accordance with the hydrodynamic loading and the dimensionless analyses, a series of pipe-soil interaction tests have been conducted with an updated pipe-soil interaction facility including a load-displacement synchronous measurement system, to reveal the underlying pipe-soil interaction mechanism. The effects of pipe surface roughness, end-constraint and initial embedment are investigated, respectively. The values of lateral-soil-resistance coefficient for the rough pipes are bigger than those for the smooth pipes. For a fixed value of non-dimensional submerged weight, the values of lateral-soil-resistance coefficient for the anti-rolling pipes are much larger than those for the freely laid pipes. The effects of initial embedment on the ultimate soil resistance get less with the decrease of the submerged weight of the pipe. A comparison is made between the results of the present mechanical-actuator tests and those of the previous water-flume tests, indicating that those results are quite comparable. For the equivalent level of dimensionless submerged weight, the directly laid pipe in currents has higher lateral stability than in waves.
\end{abstract}

(c) 2010 Elsevier Ltd. All rights reserved.

\section{Introduction}

The submarine pipeline on-bottom stability in the severe ocean environments involves a complex pipe-soil interaction process. To avoid the occurrence of pipeline on-bottom instability, i.e. the breakout of the pipe from its original site, the seabed must provide enough soil resistance to balance the hydrodynamic loads upon the untrenched pipeline. When a pipeline is laid on the seabed, e.g. during installation as well as in the operational phase, its on-bottom stability is largely related to the interaction between the pipeline and the neighboring soil. For pipeline geotechnical engineers, one of the main concerns for pipeline on-bottom stability design is to properly determine the ultimate soil resistance in severe ocean environments (Det Norske Veritas, 2007).

In the past few decades, the pipe-soil interactions have attracted much interest from pipeline researchers and designers. Before 1970s, Coulomb friction theory was employed to estimate the friction force between pipeline and soil under the action of ocean waves. Actually, Coulomb friction theory is far from the realistic pipe-soil interaction. Lyons (1973) experimentally

\footnotetext{
This manuscript was submitted to Ocean Engineering-Special Issue (Offshore Geotechnics), for review on June 3, 2010.

* Corresponding author. Tel.: +8610 82544189; fax: +861062561284.

E-mail address: fpgao@imech.ac.cn (F.-P. Gao).
}

explored the wave-induced stability of the untrenched pipeline, and concluded that the Coulomb friction theory was not suitable to describe the wave-induced interaction between pipeline and soil. In the 1980s, a few large projects had focused particularly on solving wave-induced pipe-soil interaction problems, such as the PIPESTAB project (Wagner et al., 1989), the AGA project (Brennodden et al., 1989) and a project at DHI (Palmer et al., 1988). Numerous experimental studies on the lateral stability of untrenched pipelines have been previously carried out with a mechanical-actuator simulation method. Among these, Wagner et al. (1989) improved the Coulomb friction theory into an empirical pipe-soil interaction model, in which the total lateral resistance was assumed to be the sum of the Coulomb friction component and the soil passive resistance component. Brennoden et al. (1989) further proposed an energy-based pipe-soil interaction model, in which the soil passive resistance component is related to the work done by the pipe during its movement. Using the PIPESTAB, AGA and DHI experimental data, Verley and Sotberg (1992) and Verley and Lund (1995) developed pipe-soil interaction models on sandy and clay soils, respectively, taking into account of the penetration effects of the pipe subjected to oscillatory forces in waves. Zhang et al. (2002) conducted a series of centrifugal tests to investigate the pipe-soil interaction for a shallowly embedded pipeline in calcareous sand. Foray et al. (2006) studied the pipe-soil interaction with special emphasis on 
the conditions leading to liquefaction around a pipe. White and Cheuk (2008) investigated the soil resistance on seabed pipelines during large cycles of lateral movement. The aforementioned studies focused mainly on the wave-induced pipeline on-bottom stability, in which some kinds of cyclic mechanical-actuators were employed for the simulation of wave loads on the pipe. Recently, a series of water flume tests were made to further reveal the flowpipe-soil coupling effects on the wave-induced pipe lateral instability (e.g., Gao et al., 2003; Teh et al., 2003).

With the oil and gas exploitation moving into deeper waters, ocean current becomes the prevailing hydrodynamic load for onbottom stability of submarine pipelines (Jones, 1985). Although the pipe on-bottom stability in currents seems less complex than in waves, till now, the underlying physical mechanism has not been well revealed (Jones, 1978; Gao et al., 2007). The ocean current-induced pipeline instability has been investigated by Gao et al. (2007) with small-scale water flume tests. Recently, numerical method was also adopted by a few researchers to investigate the pipe-soil interaction mechanisms for the onbottom stability of partially embedded pipes for various loading conditions, e.g., the wave loading (Takatani, 2005) and the oceancurrent loading (Gao et al., in press).

This paper aims to explore the physical mechanism of pipeline on-bottom stability in ocean currents. The ultimate lateral soil resistances to the partially embedded pipes with two kinds of constraint conditions, i.e. freely laid pipes and anti-rolling pipes, are studied experimentally. Furthermore, a comparison is made between the results of the mechanical actuator experiments and those of the previous water-flume tests.

\section{Dimensional analyses for current-induced pipeline instability}

The ocean current induced pipeline on-bottom stability on a sandy seabed is an interaction between the flow, pipe and soil. The ultimate lateral soil resistance $\left(F_{u}\right)$ is mainly related to the following characteristic parameters of the pipe, the soil and the hydrodynamic load

$F_{u}=f\left(W_{S}, D, k, \rho_{s a t}, \rho_{w}, d_{s}, D_{r}, \phi, g, \tan \theta, e_{0}, \lambda, \ldots\right)$

where $W_{S}$ is the submerged weight of the pipeline per meter; $D$ the outer diameter of pipeline; $k$ the roughness of the pipe surface; $\rho_{\text {sat }}$ the mass density of saturated sand; $\rho_{w}$ the mass density of fluid; $d s$ the diameter of sand particles; $D_{r}$ the relative density of sand; $\phi$ the internal frictional angle of sand; $g$ the gravitational acceleration; $\tan \theta$ the ratio of the lift (vertical) and the drag (horizontal) force on the pipe (see Fig. 1); $e_{0}$ the initial settlement of the pipe, which is a deduced (not independent) variable if the pipe settles into the soil under its submerged weight; and $\lambda$ represents the end constraint conditions of the pipe. In this study, two kinds of end constraints are taken into account, i.e. freely laid pipes and anti-rolling pipes.

Based on the Buckingham Pi-Theorem in the dimensional analysis theory, the dimensionless variables can be obtained from Eq. (1) as follows:

$\eta=f^{\prime}\left(G, k / D, \rho_{\text {sat }} / \rho_{w}, D / d_{s}, D_{r}, \phi, \tan \theta, e_{0} / D, \lambda, \ldots\right)$

where the lateral-soil-resistance coefficient $(\eta)$ is defined as

$\eta=\frac{F_{u}}{W_{S}-F_{u} \tan \theta}$

whose physical meaning is the ratio of the ultimate lateral soil resistance $\left(F_{u}\right)$ to the vertical pipe-soil contact force $\left(W_{S}-F_{u} \tan \theta\right) ; G$ is the non-dimensional submerged weight of

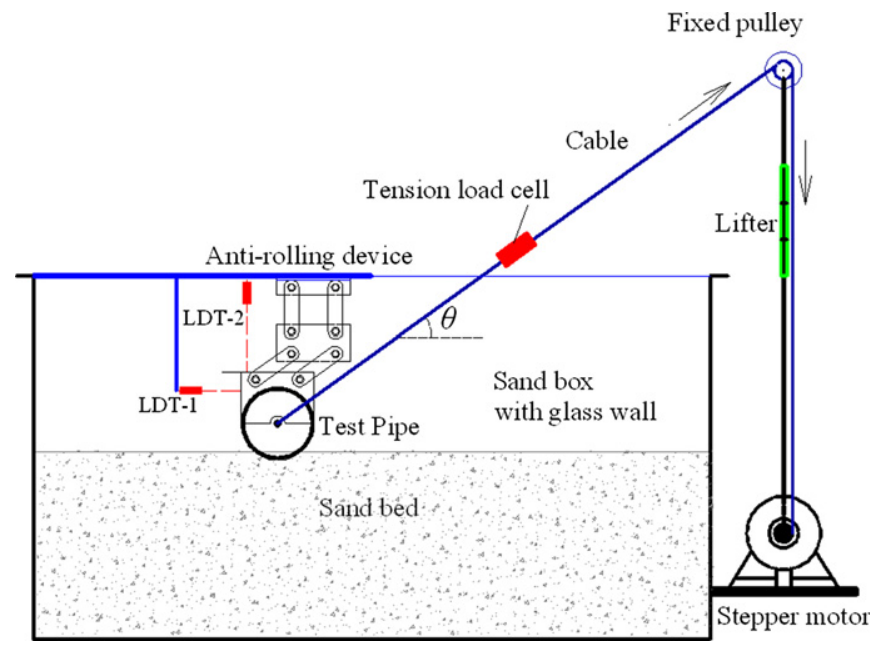

Fig. 1. Schematic diagram of the displacement-controlled experimental setup for pipe-soil interaction.

the pipe

$G=\frac{W_{S}}{\gamma^{\prime} D^{2}}$

where $\gamma^{\prime}=\left(\rho_{\text {sat }}-\rho_{w}\right) g$ is the buoyant unit weight of the saturated sand; $k / D$ the relative roughness of the pipe surface; $\rho_{\text {sat }} / \rho_{w}$ the specific gravity of the saturated sand, i.e. the ratio of the density of the saturated sand to that of the pore water; $D / d_{s}$ the ratio of pipe diameter to sand diameter; and $e_{0} / D$ the dimensionless initial settlement.

In this study, a kind of saturated sand-bed was adopted, whose index properties are given in Section 3.3, thus the values of the dimensionless parameters $\rho_{\text {sat }} / \rho_{w}, D_{r}, \phi$ (see Eq.(2)) keep constant. Three values of the pipe diameter were chosen $(D=0.10,0.15$ and $0.20 \mathrm{~m})$, so $D / d_{s}$ is approximately in the range from 250 to 500 , indicating the characteristic size of the pipes is prevailingly larger than that of the sand particles. The inclination angle $(\theta)$ of the exerted loads is approximately between $53^{\circ}$ and $57^{\circ}$ in the tests, thus values of the ratio of the lift and the horizontal drag force on the pipe (i.e. $\tan \theta$ ) is about $1.4 \pm 0.1$. In Section 4, the effects of the relative roughness of the pipe surface $(k / D)$, the end constraint conditions of the pipe (represented as $\lambda)$ and the dimensionless initial settlement $\left(e_{0} / D\right)$ on the pipe lateral stability will be discussed, respectively.

\section{Mechanical-actuator simulation of pipe-soil interaction}

\subsection{Hydrodynamic loads on submarine pipeline in currents}

To efficiently simulate the ocean currents induced hydrodynamic loads upon a submarine pipeline is crucial for evaluating pipeline lateral on-bottom stability. According to Morison's equation, the horizontal and lift (vertical) components of the steady flow-induced hydrodynamic forces can be written as follows (Morison et al., 1950):

$F_{D}=0.5 C_{D} \rho_{w} D U^{2}$

$F_{L}=0.5 C_{L} \rho_{w} D U^{2}$

where $F_{D}$ is the horizontal drag force, $F_{L}$ the vertical lift force, $C_{D}$ the drag coefficient, $C_{L}$ the lift coefficient and $U$ the effective water particle velocity. Variations of the drag and lift coefficients, $C_{D}$ and $C_{L}$, with the Reynolds number (Re) for various values of pipe surface roughness have been obtained by Jones (1978). As shown 
a

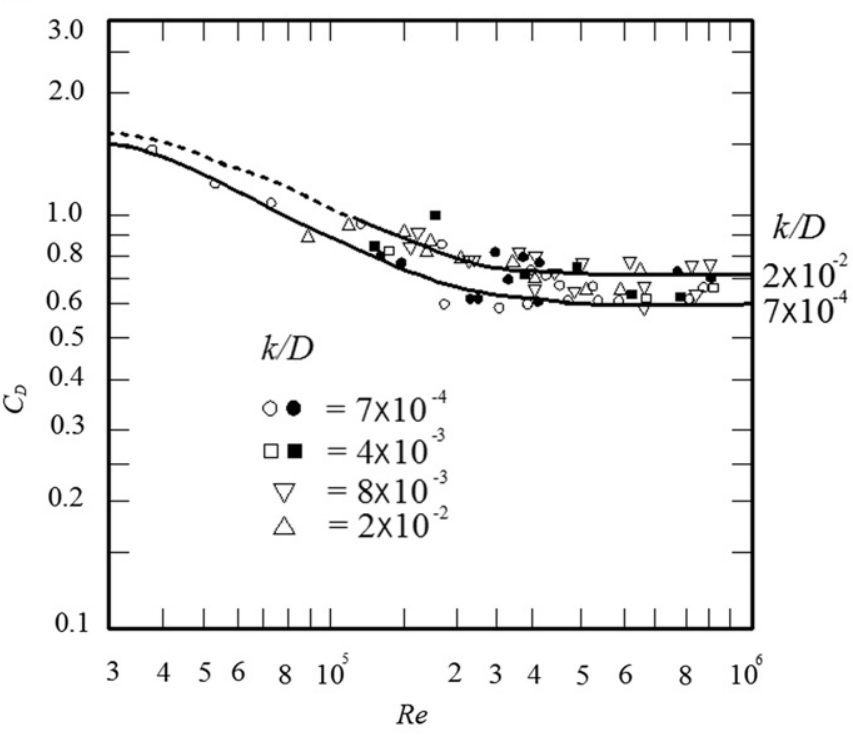

b

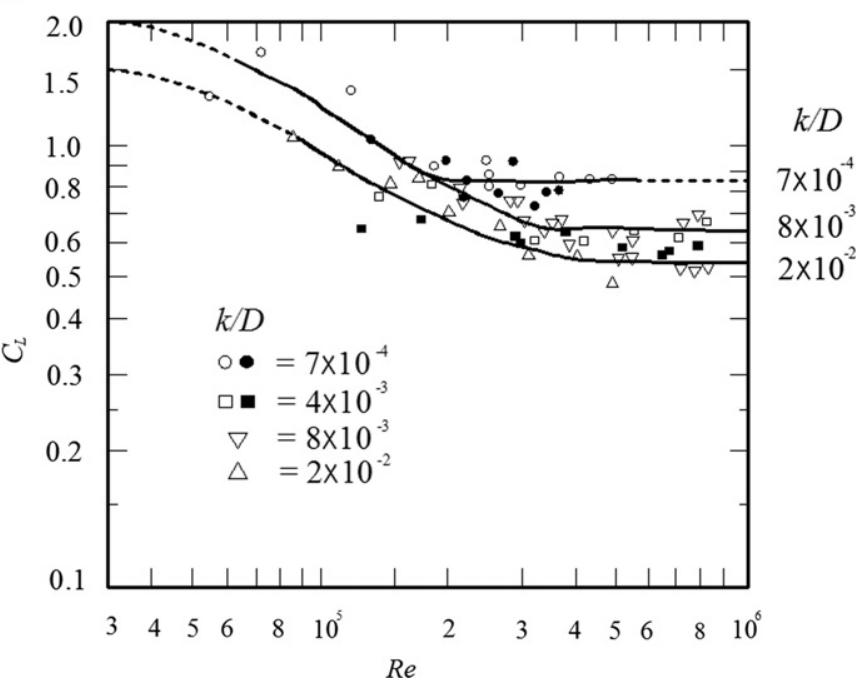

Fig. 2. Effective hydrodynamic coefficients for a pipe resting on the sea bottom recommended by Jones (1978): (a) drag coefficient and (b) lift coefficient.

in Fig. 2, with Re increasing from $3.0 \times 10^{4}$ to $1.0 \times 10^{6}$, both the drag coefficient $C_{D}$ and the lift coefficient $C_{L}$ decrease gradually to constant values with similar trends. The resultant hydrodynamic load upon the pipe is obliquely upwards with the inclination angle

$\theta=\arctan \left(F_{L} / F_{D}\right) \approx \arctan \left(C_{L} / C_{D}\right)$

Referring to the experimental results for the case of $k / D=7.0 \times 10^{-4}$ (see Fig. 2), the inclination angle $(\theta)$ is approximately between $53^{\circ}$ and $57^{\circ}$.

\subsection{Mechanical-actuator test setup and testing method}

For a pipeline laid on the seabed under the action of ocean currents, there exists a dynamic balance between the submerged weight of the pipe, the hydrodynamics forces (including the horizontal drag force $F_{D}$ and the vertical lift force $F_{L}$ ) and the soil resistances (including the lateral soil resistance $F_{H}$ and the vertical supporting force). When the ultimate lateral soil resistance is not able to balance the horizontal drag force, the pipe would breakout from its original site, i.e. the lateral instability occurs.

As aforementioned, the mechanical actuator simulation method has ever been employed in the previous studies, but which were mainly for wave-induced pipeline on-bottom stability. An updated experimental facility has recently been designed and constructed, as depicted in Fig. 1.

The facility mainly consists of a sand box with glass wall, a mechanical-actuator, the measurement system, etc. (see Fig. 1). In the sand box ( $2.0 \mathrm{~m}$ long, $0.5 \mathrm{~m}$ wide and $0.6 \mathrm{~m}$ deep), a saturated sand-bed with certain relative density was prepared by employing the sand-raining technique. In the testing process, the sands in the box were kept saturated with approximately $2 \mathrm{~cm}$ depth of free water on the surface. In the mechanical-actuator system, a displacement-controlled testing program was adopted. A stepper motor generated an inclined force onto the test pipe via a cable passing through a fixed pulley, for simulating steady currents induced drag force and lift force on the pipeline. Meanwhile, a lifter was used to adjust the inclination angle, which was maintained in the range $53-57^{\circ}$ according to the above analyses. In the synchronous measurement system, two laser displacement transducers (LDT-1 and 2, see Fig. 1) were employed for the noncontact measurement of pipe displacements, i.e. LDT- 1 is for the horizontal displacement of the test pipe, and LDT-2 for vertical settlement; meanwhile, a tension load cell was used for the measurement of the exerted inclined loads onto the test pipe. In the process of experiments, the pipe displacements and the exerted forces were measured simultaneously. The movement of the pipe was also recorded concurrently through the transparent glass wall with a digital video camera.

The testing procedure was adopted as follows: (1) the test pipe was laid downward to the soil surface. The laser displacement transducer (LDT-2) was triggered while the pipe touching at the sand-bed surface; (2) the pipe then settled into the sand-bed due to its submerged weight; meanwhile the initial settlement of the pipe was measured with LDT-2; (3) after the initial settlement finished, the stepper motor was started to impose an inclined load onto the pipe via a cable passing through a fixed pulley, for simulating steady currents induced hydrodynamic forces. During the pipe breakout process, the pipe additional settlements and the corresponding loads were measured simultaneously. The phenomena were recorded with a digital video camera through the transparent side wall.

\subsection{Parameters of test sandy soils and the test pipes with two kinds} of constraints

Three values of the diameter of pipelines with various submerged weights were used, i.e. $D=0.10,0.15$ and $0.20 \mathrm{~m}$, respectively. Two values of surface roughness of the test pipes were examined, i.e. $k=1.06 \times 10^{-4} \mathrm{~m}$ (note: $k / D \approx 6.0 \times 10^{-4}$ for $D=0.15-0.20 \mathrm{~m}$ ), $1.25 \times 10^{-5} \mathrm{~m}$ (note: $k / D \approx 7.0 \times 10^{-5}$ for $D=0.15-0.20 \mathrm{~m})$. All of the test pipes are $0.48 \mathrm{~m}$ long, with $10 \mathrm{~mm}$ gaps to the side walls.

As to a long-distance laid pipeline, the stability of pipeline at separate sections is different. For example, the demand for the stability of pipeline sections near risers is much higher than that of normal sections. Due to the constraint from risers and pipeline's own anti-torsion rigidity, the pipeline movement is neither purely parallel nor purely rotational. As such, the following two constraint conditions are considered:

Case I: Anti-rolling pipes. Pipe's rolling is restricted, but the pipe can move freely in horizontal and vertical directions. An antirolling device was designed, with which the test pipe was attached to the two side walls of the sand box (see Fig. 1). 


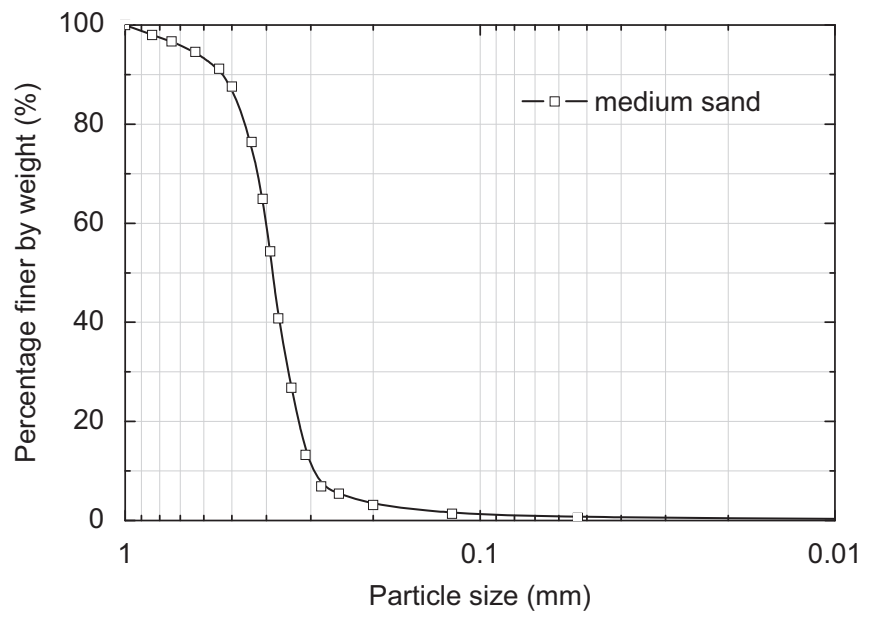

Fig. 3. Particle size distribution curve of the test sand.

Table 1

Index properties of the test sands.

\begin{tabular}{llllllll}
\hline Sand type & $d_{50}(\mathrm{~mm})$ & $d_{10}(\mathrm{~mm})$ & $C_{u}$ & $\gamma^{\prime}\left(\mathrm{kN} / \mathrm{m}^{3}\right)$ & $\phi$ (deg.) & $n$ & $D_{r}$ \\
\hline Medium sand & 0.38 & 0.30 & 1.4 & 9.30 & 26.7 & 0.73 & 0.37 \\
\hline
\end{tabular}

Case II: Freely laid pipes. The test pipe may rotate around its axis without any end constraint. In this case, the anti-rolling device does not function.

A kind of medium silica sand was adopted to simulate a sandy seabed, whose grain size distribution is shown in Fig. 3. The saturated sand-bed was prepared in the soil box by employing the sand-raining technique, whose surface was trimmed horizontally smooth with a manual scraper. The difference of the unit weights for various tests was controlled within the error of 5\%. The index properties of the sand are shown in Table 1 , where $d_{50}$ is the mean particle diameter; $d_{10}$ defined as the effective size of the particle, the grain size at which $10 \%$ of the soil weight is finer; $C_{u}$ the uniformity coefficient $\left(C_{u}=d_{60} / d_{10}\right) ; \gamma^{\prime}$ the buoyant unit weight; $\phi$ the internal friction angle; and $D_{r}$ the relative density of the sand.

\section{Experimental results and discussions on pipe-soil interaction mechanism}

\subsection{Typical pipe-soil interaction process for the pipe losing lateral stability}

Fig. 4(a) illustrates the typical experimental results on the development of lateral soil resistance $\left(F_{H}\right)$ and the corresponding vertical pipe-soil contact force $\left(W_{S}-F_{H} \tan \theta\right)$ for the anti-rolling pipes (Case I) with various values of submerged weight (i.e., $W_{S}=0.080,0.225$ and $0.305 \mathrm{kN} / \mathrm{m}$ ) during losing lateral on-bottom stability. With the increase of horizontal displacement $\left(S_{X}\right)$ during the pipe losing lateral stability, the horizontal lateral soil resistance $\left(F_{H}\right)$ increases gradually to its maximum value when the additional settlement is nearly fully developed according to the experimental observation. Meanwhile, the corresponding vertical pipe-soil contact force $\left(W_{S}-F_{H} \tan \theta\right)$ decreases gradually to its minimum value.

Fig. 4(b) shows the variation of pipe settlement with its horizontal displacement of the anti-rolling pipes while losing lateral stability. In this figure, $e_{y}$ is the settlement of the moving pipe, the negative sign means the settlement direction is downward. As shown in Fig. 4(b), the values of the initial embedment increases with increasing pipe self-weight; While the pipe breaking out from its original site (or with increasing horizontal displacement of the pipe), some additional settlement might be further developed. When the horizontal displacement $S_{x}$ is approximately $0.25-0.50 D$, the total settlement reaches its maximum value.

Experimental observation also indicates that the breakout of anti-rolling pipe was always accompanied by forming a sand wedge due to the collection of sand particles in front of the moving pipe. That is, the buildup of the ultimate lateral soil resistance to the anti-rolling pipe benefits from both the additional settlements and the sand-particle collections.

The typical characteristics for the lateral stability of freely laid pipes were also investigated. Fig. 5 gives the comparison of the variation of lateral soil resistance with the horizontal displacement between the two pipe end constraints, i.e. Case I: anti-rolling pipes and Case II: freely laid pipes. As shown in Fig. 5, for both the Cases, the lateral soil resistance increases to its maximum value with increasing horizontal displacement of the pipe. Nevertheless, the ultimate lateral resistance for the anti-rolling pipe is much larger than that for the freely laid pipe for a fixed value of pipe submerged weight $(W s)$. The increments of the ultimate lateral resistance due to the effects of end constraint increase with the increase of Ws. Experimental observation also shows that, without the anti-rolling constraint, the freely laid pipe rotated along the soil surface, without obvious collection of the neighboring sand particles in front of the shallowly embedded pipe while losing lateral stability.

\subsection{Effects of pipe surface roughness $(k / D)$}

As indicated in the aforementioned dimensional analysis, the pipe surface roughness may have influences on the pipe-soil interactions. To this aim, two values of pipe surface roughness were considered, i.e. $k=1.06 \times 10^{-4} \mathrm{~m}, \quad\left(k / D \approx 6.0 \times 10^{-4}\right.$ for $\left.D=0.15-0.20 \mathrm{~m}\right)$, $1.25 \times 10^{-5} \mathrm{~m}\left(k / D \approx 7.0 \times 10^{-5}\right.$ for $\left.D=0.15-0.20 \mathrm{~m}\right)$.

Fig. 6 shows the experimental results of the effects of pipe surface roughness on the development of pipe settlement and the corresponding lateral soil resistance during an anti-rolling pipe breaking out from its original site. Fig. 6(a) shows that, the initial embedment for the rougher pipe $\left(e_{0} / D=0.013\right.$ for the pipe with $k / D \approx 6.0 \times 10^{-4}$ ) is smaller than that for the smoother pipe $\left(e_{0} / D=0.022\right.$ for $\left.k / D \approx 7.0 \times 10^{-5}\right)$. Nevertheless, the maximum embedment in the breakout process for the rougher pipe $\left(e_{m} / D=0.050\right.$ for $\left.k / D \approx 6.0 \times 10^{-4}\right)$ is bigger than that for the smoother pipe $\left(e_{m} / D=0.041\right.$ for $\left.k / D \approx 7.0 \times 10^{-5}\right)$. The ultimate lateral resistance is increased correspondingly with the increase of the maximum embedment (see Fig. 6(a) and (b)).

A series of tests have been conducted for the two values of pipe surface roughness (see Fig. 7). As shown in Fig. 7, for a certain value of dimensionless pipe weight $(G)$, the lateral-soil-resistance coefficient $(\eta)$ for the rough pipe $\left(k / D \approx 6.0 \times 10^{-4}\right)$ is bigger than that for the smooth pipe $\left(k / D \approx 7.0 \times 10^{-5}\right)$. The dependency of $\eta$ on $G$ is ignorable for the smooth pipes.

\subsection{Effects of pipe end constraints $(\lambda)$}

Fig. 8 shows the influence of end constraint conditions $(\lambda)$ on the maximum settlement and the lateral-soil-resistance coefficient for various dimensionless submerged weights.

As indicated in Fig. 8(a), for both the anti-rolling pipes and the freely laid pipes, the values of maximum settlements increase with increasing the non-dimensional submerged weight of the pipe. The maximum settlements of anti-rolling pipes are 
a

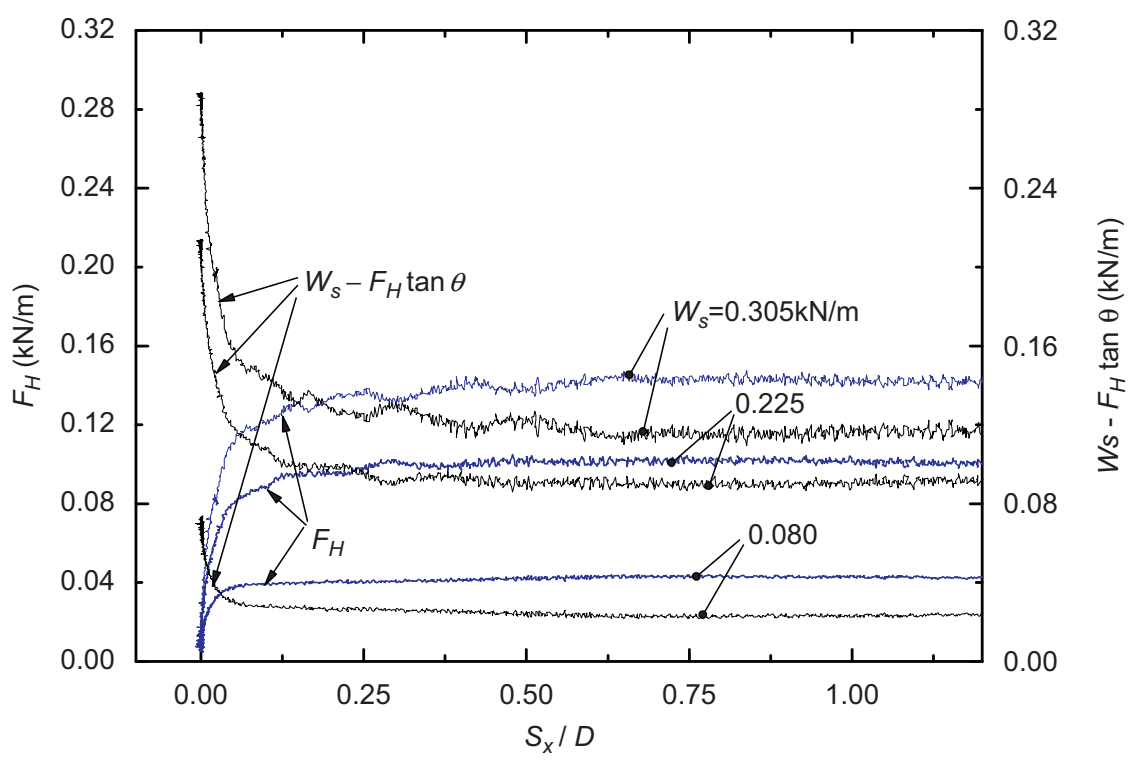

b

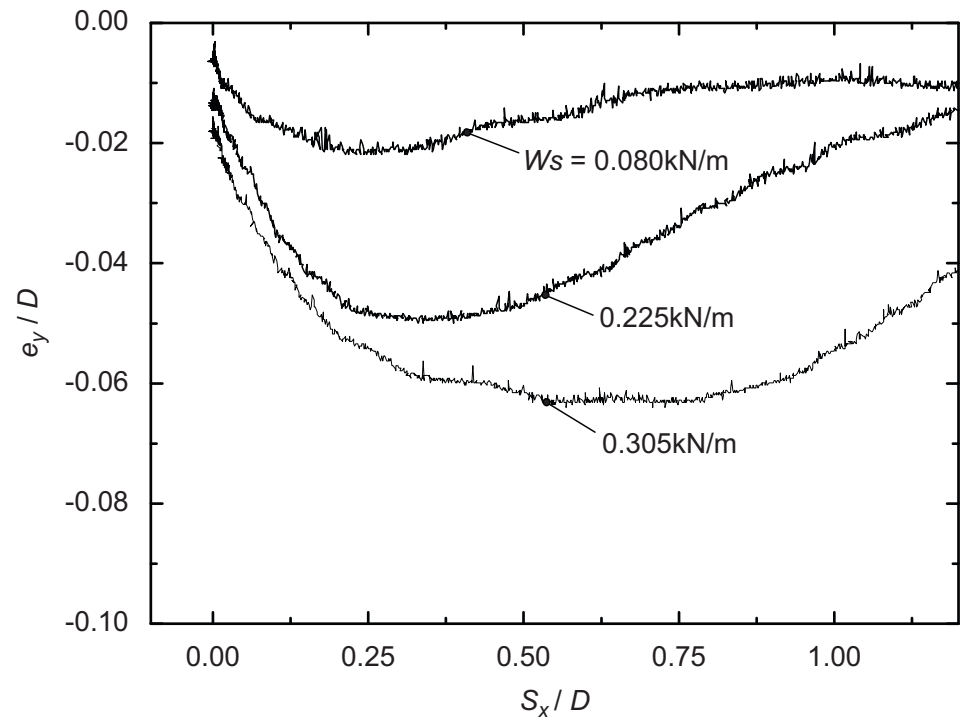

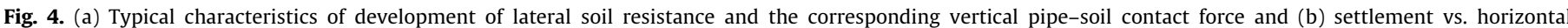

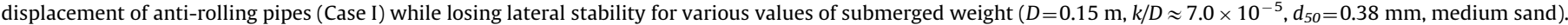

obviously bigger than those of freely laid pipes. Fig. 8(b) gives the variation of lateral-soil-resistance coefficients $(\eta)$ with the dimensionless submerged weight of the pipes $(G)$. As shown in this figure, the values of $\eta$ for anti-rolling pipes decrease slightly with the increase of $G$. For fixed values of $G$, the values of $\eta$ for the anti-rolling pipe are much larger than those for the freely laid pipe. Note that the size effect of pipe diameter on the relationship between $e_{m} / D$ and $G$ is noticeable (see Fig. 8(a)); however, its effects on the relationship between $\eta$ and $G$ are ignorable (see Fig. 8(b)).

\subsection{Effects of pipe initial embedment $\left(e_{0} / D\right)$}

For a directly laid pipe, there usually exits an initial embedment into the seabed soil due to its submerged weight. Moreover, the pipe-laying process may further cause the pipe to penetrate into the soil with certain additional embedment. In such cases, the initial embedment of the pipe is a variable, which would have influence on the pipe lateral stability.

In this section, the effects of initial embedment $\left(e_{0} / D\right)$ on the lateral stability are investigated with the test pipes with various values of $e_{0} / D$, i.e. $e_{0} / D=0.007,0.038,0.085,0.102$ and 0.143 . These test pipes have a fixed value of submerged weight $W_{S}=0.059 \mathrm{kN} / \mathrm{m}(G=0.63)$ and the diameter $D=0.10 \mathrm{~m}$. Fig. 9(a) gives the variation of dimensionless pipe settlement $\left(e_{y} / D\right)$ with horizontal displacement $\left(S_{x} / D\right)$ in the process of the pipe breaking out from its original location. It is indicated that, for the pipes with low values of initial embedment (e.g. $e_{0} / D=0.007,0.038$, see Fig. 9(a)), some additional penetration was created and the maximum embedment is located approximately at $S_{x} / D=0.25$; for the pipes with high values of initial embedment (e.g. $e_{0} / D=0.085$, 0.102 and 0.143 ), they break out obliquely upwards from their original locations, i.e. no obvious additional penetration was observed, which may be due to the strength hardening of the neighboring soils for the deeper penetration of the pipe. 


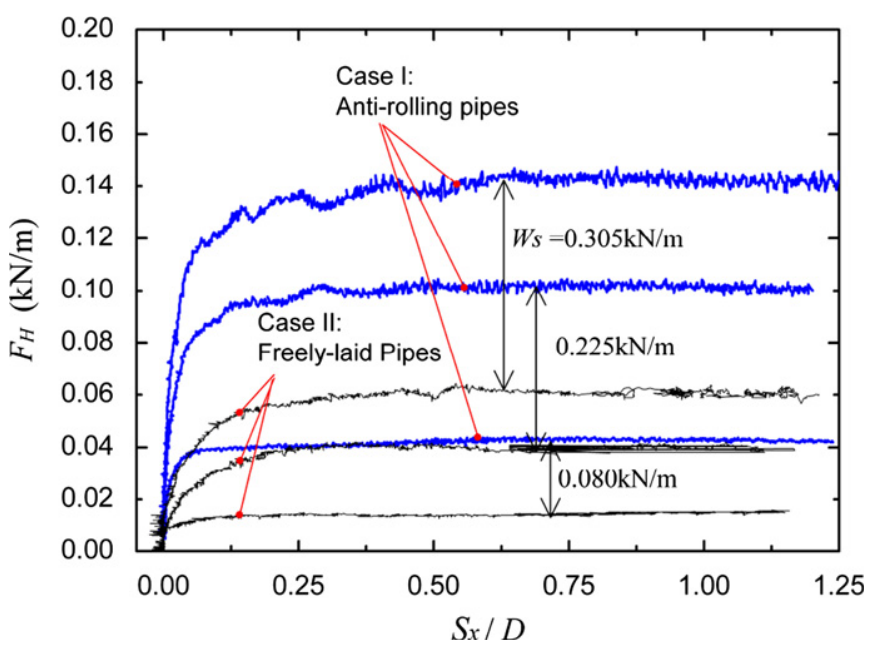

Fig. 5. Comparison of the variation of lateral soil resistance with the horizontal displacement between Case I: anti-rolling pipes and Case II: freely laid pipes $\left(D=0.15 \mathrm{~m}, k / D \approx 7.0 \times 10^{-5}, d_{50}=0.38 \mathrm{~mm}, D_{r}=0.37\right.$, medium sand $)$.
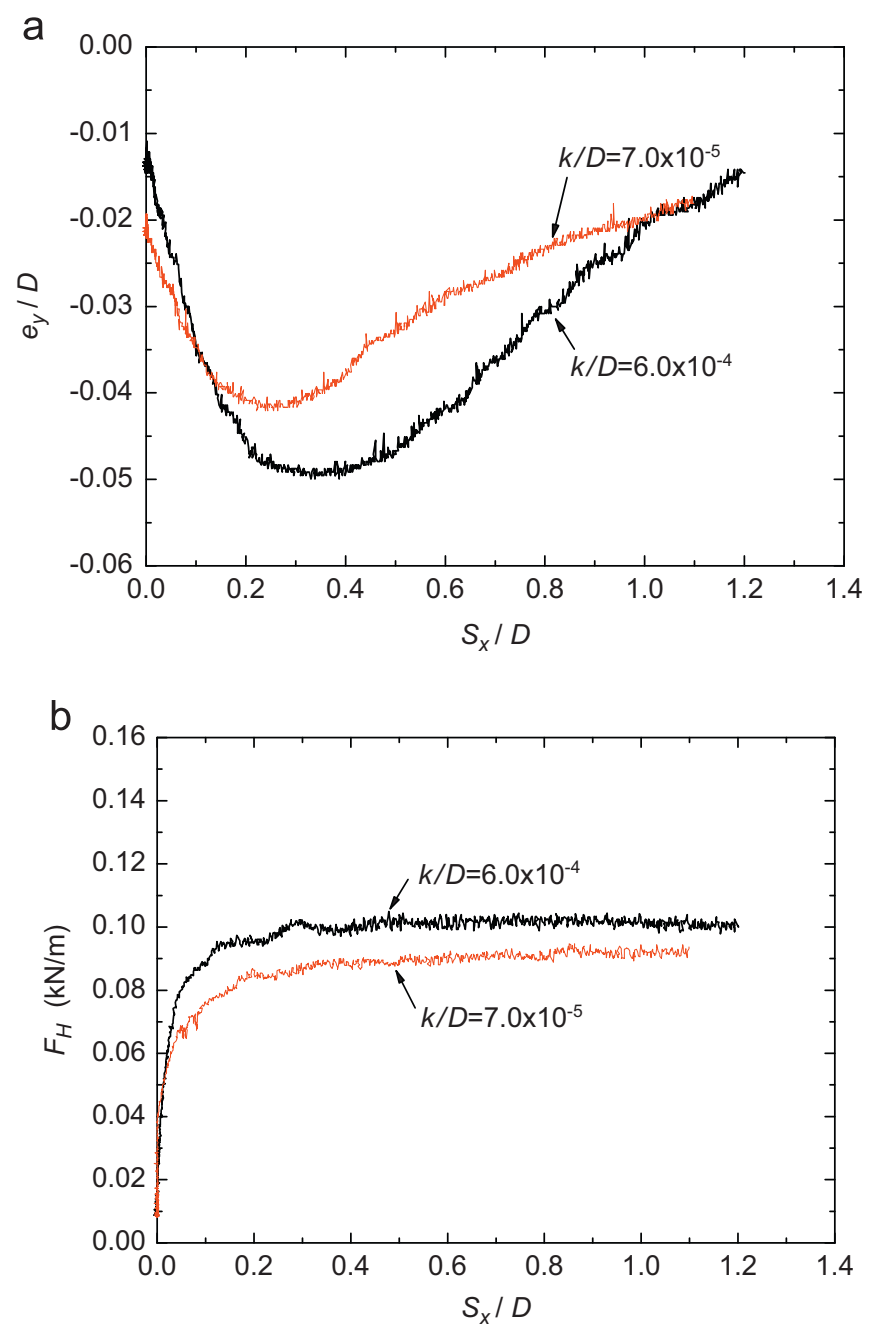

Fig. 6. Effects of pipe surface roughness on the lateral stability of anti-rolling pipes: (a) settlement vs. horizontal displacement and (b) lateral-soil-resistance vs. horizontal displacement $\left(D=0.15 \mathrm{~m}, W_{S}=0.225 \mathrm{kN} / \mathrm{m}, d_{50}=0.38 \mathrm{~mm}, D_{r}=0.37\right.$ ).

Fig. 9(b) shows the variation of the corresponding soil lateral resistance $\left(F_{H}\right)$ with the horizontal displacement $\left(S_{x} / D\right)$ of the pipe. As aforementioned, for a certain value of $e_{0} / D, F_{H}$ increases gradually

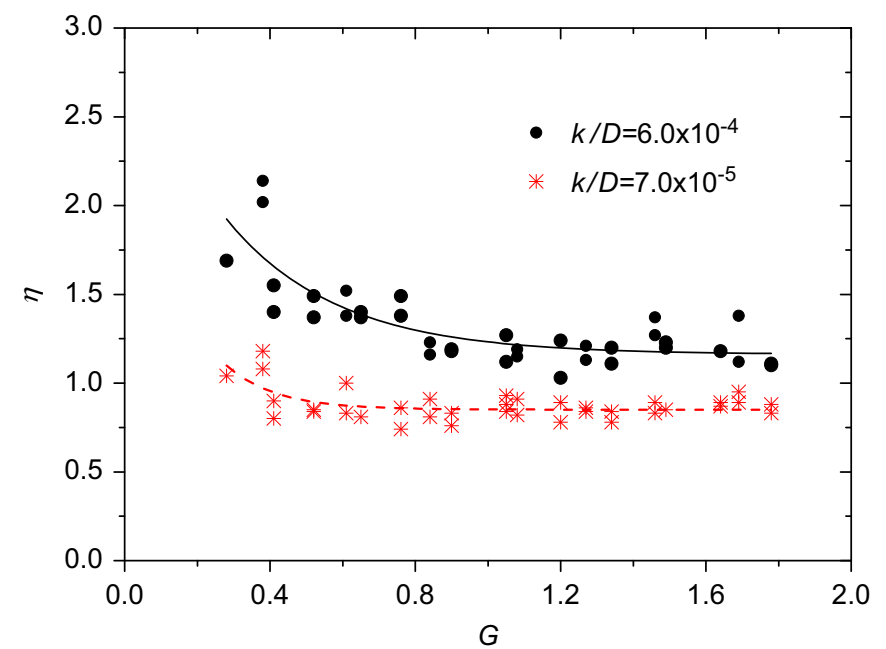

Fig. 7. Variation of lateral-soil-resistance coefficient with the dimensionless pipe weight for two values of surface roughness for anti-rolling pipes.
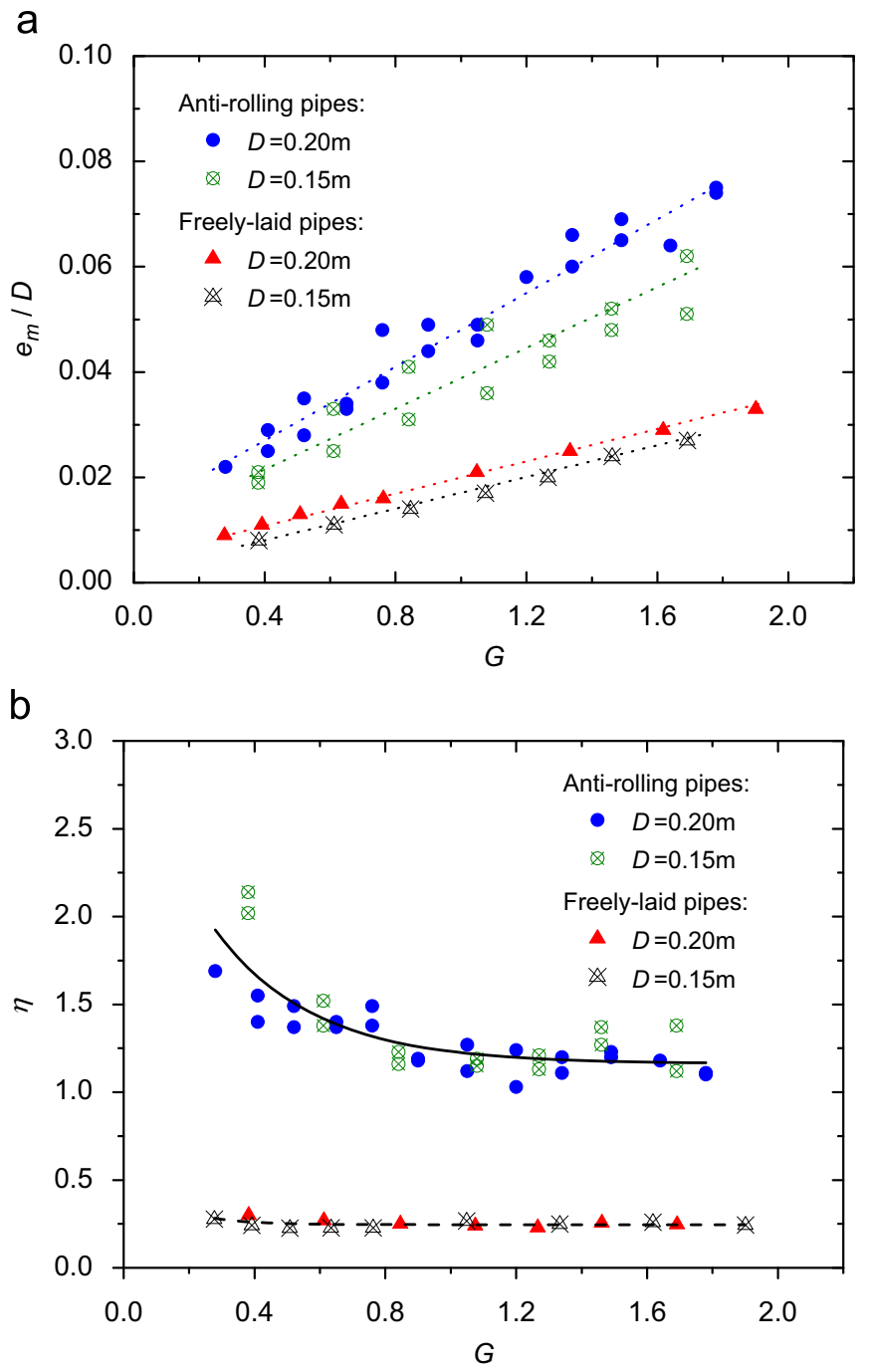

Fig. 8. Effects of end constraints on the pipe lateral stability: (a) maximum settlement $\left(e_{m} / D\right)$ vs. non-dimensional pipe weight $(G)$ and (b) lateral-soilresistance coefficient $(\eta)$ vs. non-dimensional pipe weight $(G)\left(k / D \approx 6.0 \times 10^{-4}\right.$, $\left.d_{50}=0.38 \mathrm{~mm}, D_{r}=0.37\right)$. 
a

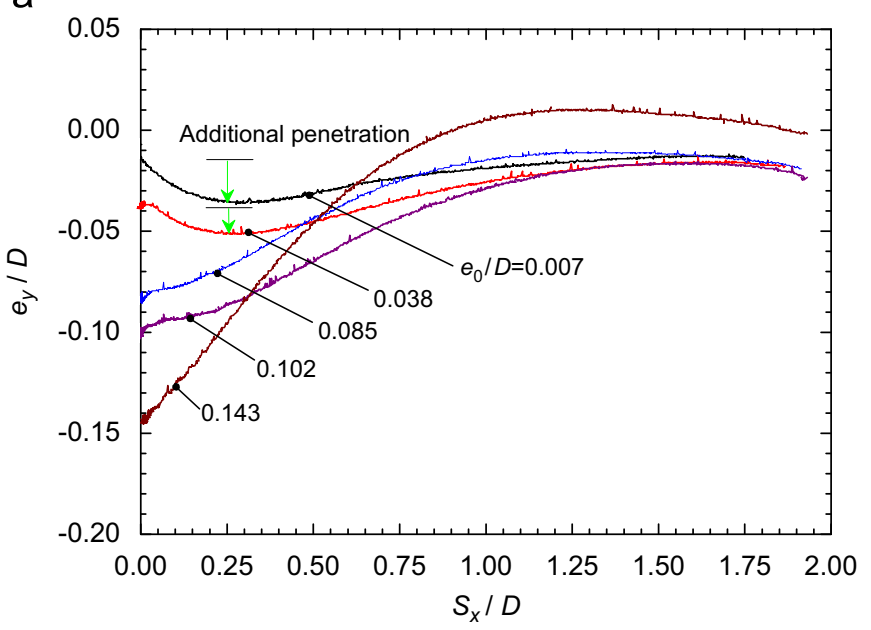

b

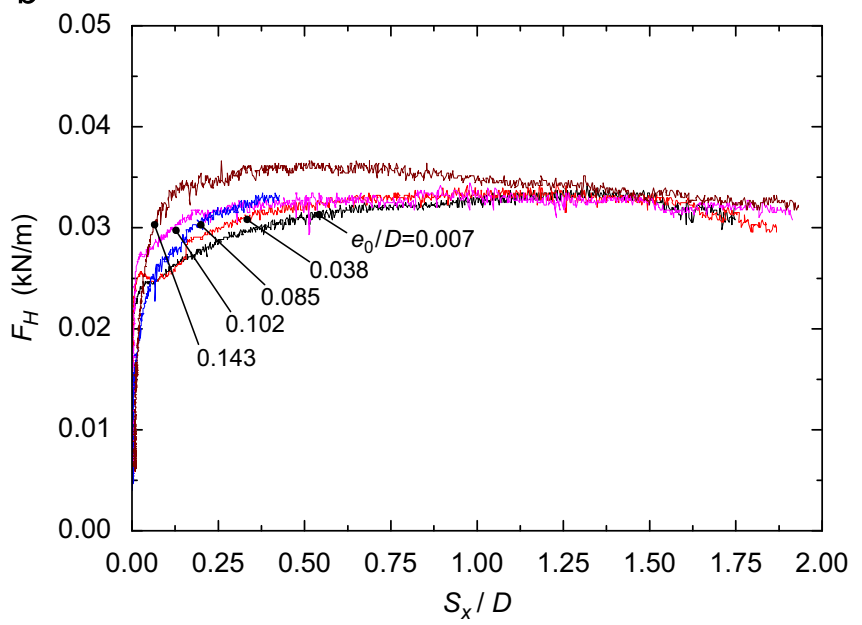

Fig. 9. Effects of initial embedment on the on-bottom stability of anti-rolling pipes: (a) variation of dimensionless pipe settlement with horizontal displacement of the pipe and (b) variation of lateral soil resistance with horizontal displacement of the pipe $\left(D=0.10 \mathrm{~m}, k / D=1.06 \times 10^{-3}, \quad W_{S}=0.059 \mathrm{kN} / \mathrm{m}\right.$, medium sand).

to its maximum value $\left(F_{u}\right)$ when the horizontal displacement of the pipe increases up to about $0.25-0.5 D$. The values of $F_{u}$ increase slightly with the increase of $e_{0} / D$. The decline of soil lateral resistance is detected for the high values of initial embedment (e.g. $e_{0} / D=0.143$, see Fig. $9(\mathrm{~b})$ ).

As expected, for the pipe with same initial embedment, the difference in submerged weight of the pipe could have influence on the pipe-soil interaction mechanism. A comparison of the pipe-soil interaction test results is made between a series of tests for the two values of submerged weight, i.e. $W_{S}=0.059$ $\mathrm{kN} / \mathrm{m}(G=0.63)$ and $0.152 \mathrm{kN} / \mathrm{m}(G=1.63)$. Fig. $10(\mathrm{a})$ and (b) shows that both the initial embedment and the submerged weight have much influence on the pipe settlement and the lateral soil resistance responses. The heavier pipe (e.g. $W_{S}=0.152$ $\mathrm{kN} / \mathrm{m}$ ) tends to induce more additional settlements for the same values of initial embedment (see Fig. 10(a)). Meanwhile, the soil resistance for the heavier pipe is much larger than that for the lighter one with the similar examined range of initial embedment $\left(e_{0} / D=0.008-0.120\right.$, see Fig. $\left.10(\mathrm{~b})\right)$. It is also indicated in Fig. 10(b) that the effects of initial pipe embedment on the ultimate soil resistance for the heavy pipe (e.g., $W_{S}=0.152$ $\mathrm{kN} / \mathrm{m}$ ) get more significant than those for the light one (e.g., $\left.W_{S}=0.059 \mathrm{kN} / \mathrm{m}\right)$. a

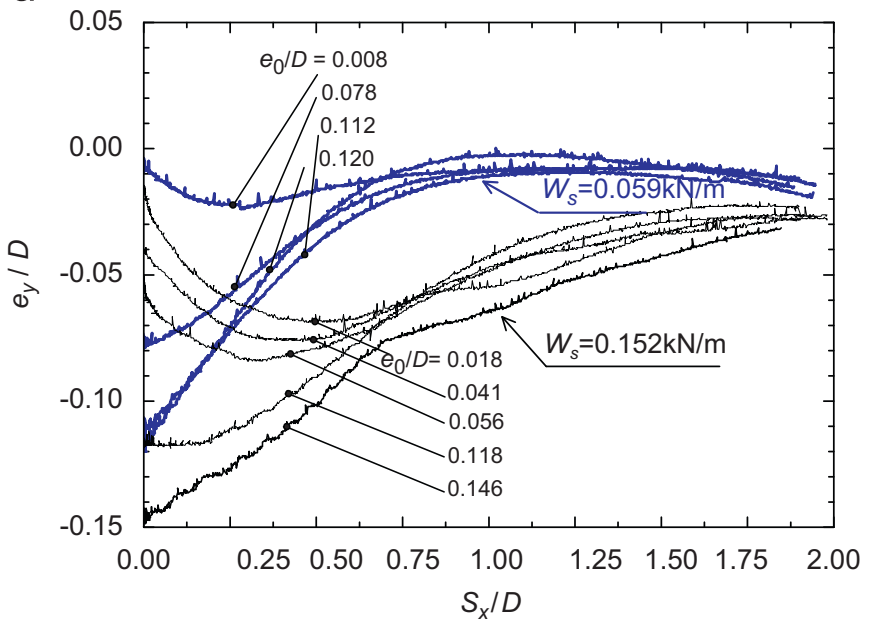

b

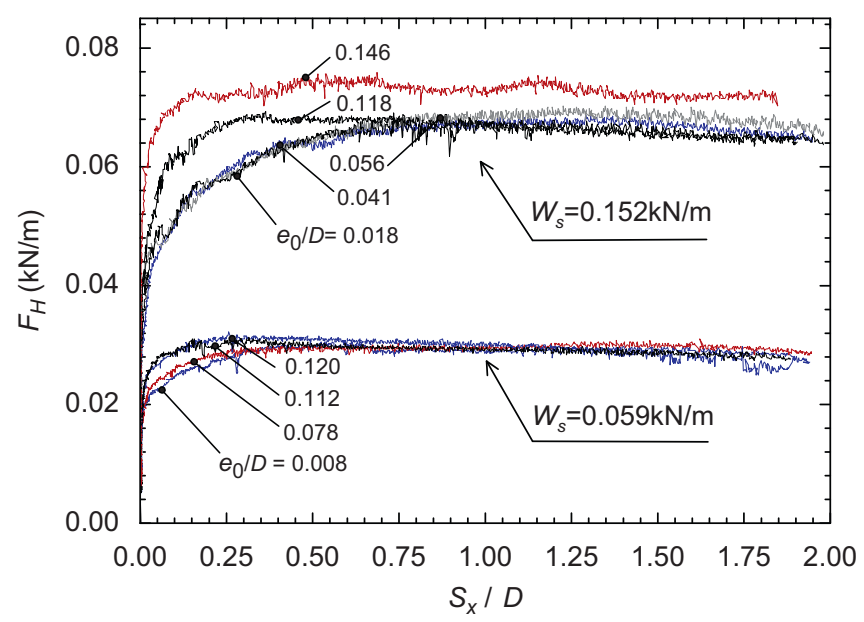

Fig. 10. Effects of initial embedment on the on-bottom stability of anti-rolling pipes for two values of pipe submerged weight $\left(W_{S}=0.059,0.152 \mathrm{kN} / \mathrm{m}\right)$ : (a) variation of dimensionless pipe settlement with horizontal displacement of the pipe and (b) variation of lateral soil resistance with horizontal displacement of the pipe $\left(D=0.10 \mathrm{~m}, k / D=1.25 \times 10^{-3}\right.$, medium sand $)$.

\section{Comparison with small-scale water flume tests}

The present mechanical-actuator simulation has the advantages for revealing the pipe-soil interaction mechanism at the prototype-scale level. The pipeline on-bottom stability had ever been investigated with water flume tests for various hydrodynamic loading conditions including waves and/or currents. It would be interesting and worthy to make a comparison between the results of the different types of tests.

The previous water flume test observation indicated that, in the process of pipeline losing lateral stability in currents, there usually exist three characteristic times: (a) onset of sand scour, (b) slight lateral displacement of pipeline and (c) breakout of pipeline (Gao et al., 2007). Based on similarity theory analyses and a series of steady-flow flume tests, Gao et al. (2007) established an empirical relationship between critical Froude number $\left(F r_{c r}\right)$ and the dimensionless submerged weight of pipe $(G)$ for describing the lateral stability of the freely laid pipes in currents. The critical Froude number $\left(\mathrm{Fr}_{\mathrm{cr}}\right)$

$F r_{c r}=U_{c r} / \sqrt{g D}$

in which $U_{c r}$ is the critical velocity of steady flow for pipe losing lateral stability. 


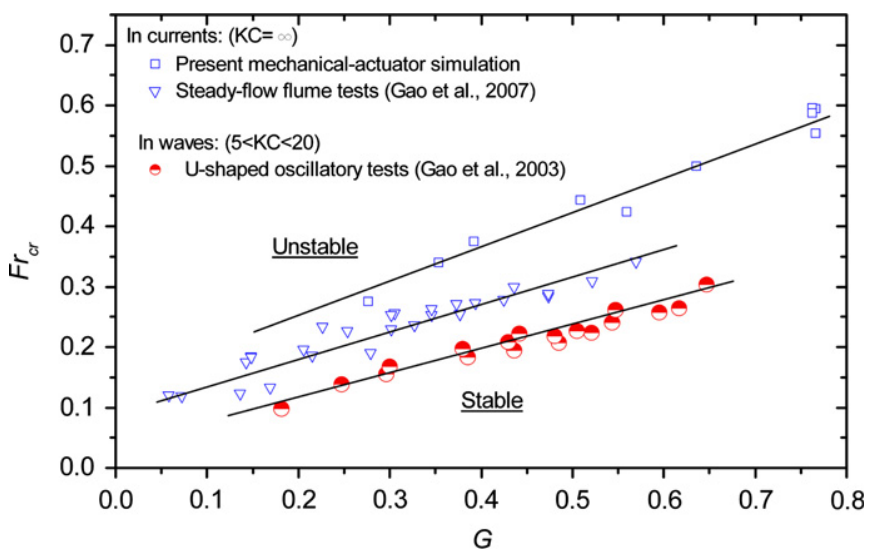

Fig. 11. Comparison of $F r_{c r}-G$ relationship between the results of present mechanical-actuator tests and previous water-flume tests.

A series of wave-induced pipeline on-bottom stability tests by Gao et al. (2003) indicated that, in the process of pipe losing on-bottom stability under the wave loading conditions, the slight rocking of the pipe is followed after the totally stable stage; after the duration of slight rocking, the pipe moves suddenly back and forth with large horizontal displacements, i.e. the pipe on-bottom instability occurs. The corresponding $\mathrm{Fr}_{c r}-\mathrm{G}$ relationship for waveinduced stability of the freely laid pipe on a sandy seabed was obtained (Gao et al., 2003). For the wave loading, the $U_{c r}$ is replaced with $U_{m c r}$, which is critical value for pipe instability of the maximum velocity of the water-particle movement induced by the waves. For the purpose of comparison of present mechanical-actuator test results with those of previous water flume tests, the corresponding $U_{c r}$ for the present tests can be calculated by submitting the ultimate lateral resistance (i.e., let $F_{D}=F_{u}$ ) into Morison's equations (see Eq. (5)).

Fig. 11 gives the comparison of $\mathrm{Fr}_{\mathrm{Cr}}-\mathrm{G}$ relationships between the results of the present mechanical-actuator tests and those of the water-flume tests by Gao et al. (2007) and of the U-shaped oscillatory flow test by Gao et al. (2003). In this figure, the parameter $\mathrm{KC}$ is the Keulegan-Carpenter number defined as $K C=U_{m} T / D$, in which $T$ is the wave period and $U_{m}$ the maximum velocity of the water-particle movement induced by the waves. In general, $\mathrm{KC}$ number controls the generation and development of vortex around pipeline, and is related to the hydrodynamic force on the pipe under wave action.

For those small-scale tests, sale effects should be examined when extrapolating their results to the real-life situation. The scale effects have been examined for the water-flume tests with the method of "modeling of models", indicating the scale effects were not obvious for the range of pipeline diameters examined and within the examined Froude range $(0.10<F r<0.35)$ (Gao et al., 2007). In the present mechanical-actuator tests, the test pipes $(D=0.10-0.20 \mathrm{~m})$ are at the prototype-scale level.

It is indicated in Fig. 11 that the experimental results for current-induced pipe lateral stability are comparable. For the same value of non-dimensional pipe submerged weight $(G)$, the values of $F r_{c r}$ for the present mechanical actuator tests are slightly greater than those for the steady-flow flume tests when increasing the non-dimensional pipe submerged weight. Note that, in the small-scale steady-flow flume tests, some local scour around the pipe was observed in the process of the pipe losing lateral stability under the action of currents. The local scour behind the untrenched pipe (down-stream) may reduce the pipeline lateral stability. A comparison has also been made between pipe lateral stability in currents and that in waves. The physical phenomena of pipeline losing lateral stability in currents are somewhat different from those in waves, especially in the stage of pipe breakout (Gao et al., 2007). As shown in Fig. 11, for the same values of the dimensionless submerged weight, the directly laid pipe in currents has higher lateral stability than in waves.

\section{Conclusions}

A series of pipe-soil interaction tests at the prototype-scale level have been conducted with an updated pipe-soil interaction facility, in order to further reveal the pipe-soil interaction mechanism for steady flow-induced on-bottom stability of a pipeline partially embedded in a sandy soil. To efficiently simulate the hydrodynamic loads on the pipe resting on the seabed, the mechanical-actuator simulation method was adopted in accordance with the hydrodynamic loading analysis. Based on the experimental observation and parametric studies, the following conclusions can be drawn:

(1) Typical pipe-soil interaction process for the pipe losing lateral stability is obtained with a load-displacement synchronous measurement system. The horizontal lateral soil resistance gradually increases and the corresponding vertical pipe-soil contact force decreases to the maximum and minimum values, respectively, which is usually accompanied with the additional settlement being developed.

(2) The steady flow-induced pipe lateral stability is affected by many influential factors, including pipe surface roughness, end-constraint, initial embedment, etc. the lateral-soil-resistance coefficient $(\eta)$ for the rough pipe is bigger than that for the smooth pipe. However, the correlation of $\eta$ with the dimensionless submerged weight $(G)$ is ignorable for the smooth pipes. For a certain value of non-dimensional submerged weight, the values of $\eta$ for the anti-rolling pipes are much larger than those for the freely laid pipes. The effects of initial embedment on the ultimate soil resistance get less with the decrease of the submerged weight of the pipe.

(3) Comparison between the results of the present mechanicalactuator tests and those of the previous water-flume tests indicates that, the results of two types of tests are comparable and the local scour in the steady flow flume reduces the pipe lateral stability. For the same values of the dimensionless submerged weight, the directly laid pipe in currents has higher lateral stability than in waves. The present test results needs further validation with future full-scale experiments or field observations.

\section{Acknowledgment}

Financial support from Knowledge Innovation Program of the Chinese Academy of Sciences (Grant no. KJCX2-YW-L02) is greatly appreciated.

\section{References}

Brennodden, H., Lieng, J.T., Sotberg, T., Verley R.L.P., 1989. An energy-based pipesoil interaction model. In: Proceedings of the 21st Annual Offshore Technology Conference, OTC 6057, pp. 147-158.

Det Norske Veritas, 2007. On-bottom stability design of submarine pipelines. Recommended Practice, DNV-RP-F109.

Foray, P., Bonjean, D., Michallet, H., Mory, M., 2006. Fluid-soil-structure interaction in liquefaction around a cyclically moving cylinder. Journal of Waterway, Port, Coastal, and Ocean Engineering 132 (4), 289-299.

Gao, F.-P., Gu, X.-Y., Jeng, D.-S., 2003. Physical modeling of untrenched submarine pipeline instability. Ocean Engineering 2003 (30), 1283-1304. 
Gao, F.-P., Yan, S.-M., Yang, B., Wu, Y.-X., 2007. Ocean currents induced pipeline lateral stability on sandy seabed. Journal of Engineering Mechanics, ASCE 133 (10), 1086-1092.

Gao, F.-P., Yan, S.-M., Zhang, E.-Y., Wu Y.-X., Jia, X.. Lateral soil resistance to an untrenched pipeline under the action of ocean currents. In: Proceedings of the Second International Symposium on Frontiers in Offshore Geotechnics (ISFOG 2010), Perth, Australia (in press).

Jones, W.T., 1978. On-bottom pipeline stability in steady water currents. Journal of Petroleum Technology 30, 475-484.

Jones, W.T., 1985. Deepwater pipeline environmental design conditions. In: Proceeding of Energy Sources Technology Conference \& Exhibition, Dallas, Texas, 1(2), pp. 450-466.

Lyons, C.G., 1973. Soil resistance to lateral sliding of marine pipeline. In: Proceedings of Fifth Annual Offshore Technology Conference, OTC 1876, pp. 479-484.

Morison, J.R., O’Brien, M.P., Johnson, J.W., Schaaf, S.A., 1950. The forces exerted by surface waves on piles. Petroleum Transactions, AIME 189, 149-157.

Palmer, A.C., Steenfelt, J.S., Jacobsen, V., 1988. Lateral resistance of marine pipelines on sand. In: Proceedings of 20th Annual Offshore Technology Conference, OTC 5853, pp. 399-408.
Takatani, T., 2005. Pipeline-seabed interaction analysis subjected to horizontal loading. In: Proceedings of the First International Symposium on Frontiers in Offshore Geotechnics (ISFOG 2005), Perth, Australia, pp. 629-635.

Teh, T.C., Palmer, A.C., Damgaard, J.S., 2003. Experimental study of marine pipelines on unstable and liquefied seabed. Coastal Engineering 50, 1-17.

Verley, R.L.P., Lund, K.M., 1995. A soil resistance model for pipeline placed on clay soils. In: Proceedings of international conference on Offshore Mechanics and Arctic Engineering (OMAE), vol. 5-A, Pipeline Technology, pp. 225-232.

Verley, R.L.P., Sotberg, T., 1992. A soil resistance model for pipeline placed on sandy soils. In: Proceedings of international conference on Offshore Mechanics and Arctic Engineering (OMAE), vol. 5-A, Pipeline Technology pp. 123-131.

Wagner, D.A., Murff, J.D., Brennodden, H., Sveggen, O., 1989. Pipe-soil interaction model. Journal of Waterway, Port, Coastal, Ocean Engineering 115 (2), 205-220.

White, D.J., Cheuk, C.Y., 2008. Modelling the soil resistance on seabed pipelines during large cycles of lateral movement. Marine Structures 21, 59-79.

Zhang, J., Stewart, D.P., Randolph, M.F., 2002. Modeling of shallowly embedded offshore pipelines in calcareous sand. Journal of Geotechnical and Geoenvironmental Engineering, ASCE 128, 363-371. 